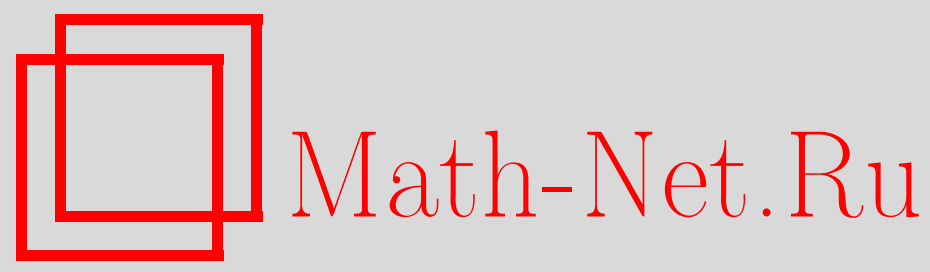

С. С. Марченков, О полиномиальной вычислимости некоторых рудиментарных предикатов, Матем. заметки, 2003, том 74, выпуск 1, 69-75

DOI: https://doi.org/10.4213/mzm246

Использование Общероссийского математического портала Math-Net.Ru подразумевает, что вы прочитали и согласны с пользовательским соглашением http://www . mathnet.ru/rus/agreement

Параметры загрузки:

IP : 54.84 .234 .179

26 апреля 2023 г., 13:46:01

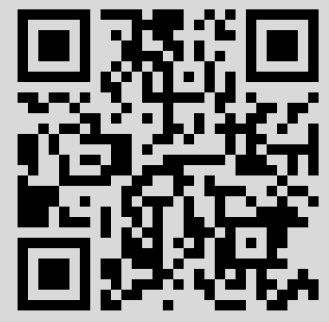




\title{
О ПОЛИНОМИАЛЬНОЙ ВЫЧИСЛИМОСТИ НЕКОТОРЫХ РУДИМЕНТАРНЫХ ПРЕДИКАТОВ
}

\author{
С. С. Марченков
}

Класс рудиментарных предикатов определяется как наименьший класс числовых предикатов, который содержит предикаты равенства и конкатенации и замкнут относительно операций логики высказываний, явных преобразований и навешивания ограниченных кванторов. Рассматриваются два класса рудиментарных предикатов. Первый класс состоит из предикатов, которые имеют в предваренной нормальной форме специального вида кванторную приставку типа $\exists \forall$. У предикатов второго класса кванторная приставка может быть произвольной, но ограничения налагаются на скулемовские разрешаюшие функции. Доказывается, что любой предикат каждого из этих классов можно вычислить подходящим детерминированньп алгоритмом за полиномиальное время.

Библиограффия: 8 названий.

Одной из распространенных форм представления числовых предикатов, часто встречающейся в теории алгоритмов, алгебре и теории чисел, является форма

$$
\left(Q_{1} y_{1}\right)_{y_{1} \leqslant z_{1}} \ldots\left(Q_{n} y_{n}\right)_{y_{n} \leqslant z_{n}} \rho\left(x_{1}, \ldots, x_{m}, y_{1}, \ldots, y_{n}\right)
$$

где $Q_{1}, \ldots, Q_{n}$ - кванторы $\exists$ или $\forall$, а предикат $\rho$ получается из арифметических предикатов $x+y=z$ и $x \cdot y=z$ с помощью операций логики высказьваний и явных преобразований (перестановка и отождествление аргументов, введение фиктивных аргументов, подстановка констант). Класс предикатов, представимых в форме (1), с небольшими техническими отличиями был определен А. В. Кузнецовым [1], как класс ограниченно-арифметических предикатов, и Р. Смальяном [2], как класс конструктивно-арифметических предикатов. В книге [2] введено также понятие рудиментарного предиката. Совпадение классов ограниченно-арифметических, конструктивно-арифметических и рудиментарных предикатов установлено в [2]-[5].

При определении рудиментарных предикатов предполагается, что натуральные числа кодируются взаимно-однозначным образом словами в алфавите $\{1,2\}$ : слову $a_{l} a_{l-1}$ $\ldots a_{1}$, составленному из символов 1 и 2 , сопоставляется натуральное число

$$
\sum_{i=1}^{l} a_{i} \cdot 2^{i-1}
$$

Работа выполнена при финансовой поддержке Российского фонда фундаментальных исследований, грант № 00-01-00351. 
Слово $a_{l} a_{l-1} \ldots a_{1}$ называется диадическим представлением числа $(2)$.

В качестве исходных рудиментарных предикатов рассматриваются предикаты равенства $x=y$ и конкатенации $x y=z$ (конкатенация диадических представлений чисел $x$ и $y$ есть диадическое представление числа $z$ ). Класс рудиментарных предикатов определяется как наименьший класс числовых предикатов, которьй содержит предикаты равенства и конкатенации и которьй замкнут относительно операций логики высказьваний, явных преобразований и навешивания ограниченных кванторов $(\exists y)_{y \leqslant z}$ и $(\forall y)_{y \leqslant z}$.

В книге [6] установлено (см. раздел III, гл. $1, \S 6$, теоремы 10, 11), что класс рудиментарных предикатов совпадает с классом всех предикатов, представимых в форме

$$
\left(Q_{1} y_{1}\right)_{y_{1} \leqslant z_{1}} \ldots\left(Q_{n} y_{n}\right)_{y_{n} \leqslant z_{n}}(T=U)
$$

а также с классом всех предикатов, представимых в форме

$$
\left(Q_{1} y_{1}\right)_{\left|y_{1}\right| \leqslant\left|z_{1}\right|} \cdots\left(Q_{n} y_{n}\right)_{\left|y_{n}\right| \leqslant\left|z_{n}\right|}(T=U)
$$

где $Q_{1}, \ldots, Q_{n}$ - кванторы $\exists$ или $\forall, T$ и $U$ - словарные термы, составленные из символов переменных и слов в алфавите $\{1,2\}$, а $|y|$ обозначает длину диадического представления числа $y$.

Попытки вычисления рудиментарных предикатов непосредственно по формулам (3) или (4) в общем случае приводят к экспоненциальному (относительно длин диадических представлений аргументов) времени вычисления. В работе [7] обнаружено, что рудиментарные $\exists$ - и $\forall$-предикаты (предикаты, в предваренной нормальной форме которых все кванторы совпадают) можно вычислить детерминированно за полиномиальное время. Дальнейшее развитие идеи, предложенной в [7], позволяет выделить еще два класса рудиментарньх предикатов, которые обладают этим свойством. Первьй класс состоит из предикатов, имеющих в представлениях (3) или (4) кванторную приставку типа $\exists \forall$ (класс рудиментарных $\exists \forall$-предикатов). Во втором классе число перемен кванторов в кванторных приставках в целом не ограничено, однако ограничения налагаются на скулемовские разрешаюшие функции (см. определения ниже). Предикаты второго класса названы в работе компактными рудиментарньми предикатами.

Хотя все формулируемые ниже результаты имеют место как при использовании представлений (3), так и при использовании представлений (4), по техническим причинам нам будет удобнее в дальнейшем иметь дело лишь с представлениями (4).

Что касается вопроса о возможном обобщении полученных в работе результатов на более широкие классы рудиментарных предикатов, то здесь необходимо сделать следующее замечание. Как установлено в работах [5], [7], средствами рудиментарных предикатов возможно моделирование недетерминированных и альтернируюших вычислений на машинах Тьюринга со входом, работающих с полиномиальным временем и с зоной, ограниченной функцией вида $l^{\gamma}$, где $0<\gamma<1$. Поэтому, например, возможность вычисления произвольных рудиментраных предикатов детерминированно за полиномиальное время представляется маловероятной.

Переходя к определениям, будем говорить, что рудиментарньй предикат $\rho$ принадлежит классу $\exists \forall$ (или является $\exists \forall$-предикатом), если $\rho$ представим в форме (4), где в кванторной приставке $Q_{1} \ldots Q_{n}$ все кванторы существования предшествуют всем кванторам обшности. 
Ниже в теоремах 1, 2 речь идет о детерминированных алгоритмах с полиномиальным временем работы. Мы описываем эти алгоритмы содержательным образом, имея в виду, что выбран достаточно широкий класс абстрактных вычислительных устройств, и оба алгоритма реализуются вычислительными устройствами этого класса. Возникающие при этом формулы, кодируются "естественньг образом" словами некоторого конечного алфавита. Все оценки времени вычисления выражаются через длины диадических представлений аргументов.

ТЕОрема 1. Любой рудиментарный предикат класса $\exists$ можно вычислить подходящим детерминированным алгоритмом за полиномиальное время.

ДокАЗАТЕЛЬСтво. Пусть рудиментарньй $\exists \forall$-предикат $\rho\left(x_{1}, \ldots, x_{m}, z_{1}, \ldots, z_{n}\right)$ представим в форме

$$
\left(\exists y_{1}\right)_{\left|y_{1}\right| \leqslant\left|z_{1}\right|} \cdots\left(\exists y_{p}\right)_{\left|y_{p}\right| \leqslant\left|z_{p}\right|}\left(\forall y_{p+1}\right)_{\left|y_{p+1}\right| \leqslant\left|z_{p+1}\right|} \cdots\left(\forall y_{n}\right)_{\left|y_{n}\right| \leqslant\left|z_{n}\right|}(T=U),
$$

где словарные термы $T, U$ составлены из символов переменных $x_{1}, \ldots, x_{m}, y_{1}, \ldots, y_{n}$ и слов в алфавите $\{1,2\}$. Введем обозначения

$$
k_{1}=\left|x_{1}\right|, \ldots, k_{m}=\left|x_{m}\right|, \quad l_{1}=\left|z_{1}\right|, \ldots, l_{n}=\left|z_{n}\right| .
$$

Очевидно, что формула (5) эквивалентна формуле

$$
\begin{aligned}
& \bigvee_{1 \leqslant i_{1} \leqslant l_{1}, \ldots, 1 \leqslant i_{p} \leqslant l_{p}}\left(\exists y_{1}\right)_{\left|y_{1}\right|=i_{1}} \\
& \ldots\left(\exists y_{p}\right)_{\left|y_{p}\right|=i_{p}}\left(\begin{array}{c}
\& \leqslant i_{p+1} \leqslant l_{p+1}, \ldots, 1 \leqslant i_{n} \leqslant l_{n} \\
\ldots
\end{array}\right)\left(\forall y_{p+1}\right)_{\left|y_{p+1}\right|=i_{p+1}} \\
& \left.\ldots\left(\forall y_{n}\right)_{\left|y_{n}\right|=i_{n}}(T=U)\right) .
\end{aligned}
$$

Для каждой из переменных $x_{s}, y_{t}(1 \leqslant s \leqslant m, 1 \leqslant t \leqslant n)$ введем множества двоичных переменных $x_{1}^{s}, \ldots, x_{k_{s}}^{s}$ и $y_{1}^{t}, \ldots, y_{i_{t}}^{t}$, значениями которых служат символы 1 и $2 . \mathrm{B}$ формуле (7) произведем замену каждой переменной $x_{s}(1 \leqslant s \leqslant m)$ блоком переменных $x_{1}^{s} \ldots x_{k_{s}}^{s}$ и каждой переменной $y_{t}(1 \leqslant t \leqslant n)$ блоком переменных $y_{1}^{t} \ldots y_{i_{t}}^{t}$. Тогда формула (7) будет эквивалентна формуле

$$
\begin{gathered}
\bigvee_{1 \leqslant i_{1} \leqslant l_{1}, \ldots, 1 \leqslant i_{p} \leqslant l_{p}}\left(\exists y_{1}^{1}\right) \ldots\left(\exists y_{i_{1}}^{1}\right) \ldots\left(\exists y_{1}^{p}\right) \ldots\left(\exists y_{i_{p}}^{p}\right)\left(\underset{1 \leqslant i_{p+1} \leqslant l_{p+1}, \ldots, 1 \leqslant i_{n} \leqslant l_{n}}{\&}\right) \\
\left.\left(\forall y_{1}^{p+1}\right) \ldots\left(\forall y_{i_{p+1}+1}^{p+1}\right) \ldots\left(\forall y_{1}^{n}\right) \ldots\left(\forall y_{i_{n}}^{n}\right)\left(T^{\prime}=U^{\prime}\right)\right),
\end{gathered}
$$

где словарные термы $T^{\prime}, U^{\prime}$ получены из словарных термов $T, U$ указанной вьше заменой переменной.

Считая фиксированньми значения $k_{1}, \ldots, k_{m}, i_{1}, \ldots, i_{n}$, проведем дальнейшие преобразования формулы $T^{\prime}=U^{\prime}$. Если $\left|T^{\prime}\right| \neq\left|U^{\prime}\right|$, то соответствующие конъюнктивный сомножитель и дизъюнктивное слагаемое формулы (8) будут ложными. Предположим, что $\left|T^{\prime}\right|=\left|U^{\prime}\right|$. Сравним тогда в термах $T^{\prime}$ и $U^{\prime}$ символы, стояшие на одинаковых позициях. Это сравнение даст систему равенств $W$, состоящую из равенств вида

$$
x_{q}^{s}=x_{r}^{t}, \quad x_{q}^{s}=y_{r}^{t}, \quad x_{q}^{s}=a, \quad y_{q}^{s}=y_{r}^{t}, \quad y_{q}^{s}=a, \quad a=b,
$$


где $a, b \in\{1,2\}$. Очевидно, что система равенств $W$ эквивалентна равенству $T^{\prime}=U^{\prime}$. Образуем далее транзитивное замыкание системы равенств $W$. Возможно, что при этом возникнут противоречивые равенства. В этом случае соответствующие конъюнктивный сомножитель и дизъюнктивное слагаемое формулы (8) будут, разумеется, ложными. Если же противоречивых равенств в транзитивном замькании не образовалось, то формула $T^{\prime}=U^{\prime}$ будет эквивалентна конъюнкции $K_{1}$ равенств вида

$$
v_{1}=\cdots=v_{d} \quad \text { или } \quad v_{1}=\cdots=v_{d}=a,
$$

где $v_{1}, \ldots, v_{d}$ - переменные типов $x_{q}^{s}, y_{r}^{t}, a \in\{1,2\}$ и различные формулы конъюнкции $K_{1}$ не имеют обших переменных.

Таким образом, после исключения случаев тож дественно ложных формул мы приходим к тому, что подформула

$$
\left(\forall y_{1}^{p+1}\right) \ldots\left(\forall y_{i_{p+1}}^{p+1}\right) \ldots\left(\forall y_{1}^{n}\right) \ldots\left(\forall y_{i_{n}}^{n}\right)\left(T^{\prime}=U^{\prime}\right)
$$

формулы (8) эквивалентна формуле

$$
\left(\forall y_{1}^{p+1}\right) \ldots\left(\forall y_{i_{p+1}}^{p+1}\right) \ldots\left(\forall y_{1}^{n}\right) \ldots\left(\forall y_{i_{n}}^{n}\right) K_{1}
$$

Согласно известным логическим правилам элиминируем в формуле (12) кванторы общности. Именно, если переменная $y_{r}^{t}$ в конъюнкции $K_{1}$ входит в равенство $(10)$, которое помимо переменной $y_{r}^{t}$ содержит еще хотя бы одну переменную либо константу, то формула (12) ложна. В остальных случаях квантор $\forall y_{r}^{t}$ в формуле (12) можно опустить (заметим, что при получении системы равенств $W$ могут появиться истинные равенства вида $y_{r}^{t}=y_{r}^{t}$, которые мы в дальнейшем не учитьваем).

Итак, всякая подформула (11) формулы (8) либо тождественно ложна, либо эквивалентна бескванторной формуле, представляющей собой конъюнкцию формул вида (10), где $v_{1}, \ldots, v_{d}$ - переменные типов $x_{q}^{s}, y_{r}^{t}, 1 \leqslant t \leqslant p$ (сюда же мы относим частный случай, когда формула (11) тождественно истинна). Поэтому аналогичное утверждение будет справедливо и для подформул

$$
\underset{1 \leqslant i_{p+1} \leqslant l_{p+1}, \ldots, 1 \leqslant i_{n} \leqslant l_{n}}{\&}\left(\forall y_{1}^{p+1}\right) \ldots\left(\forall y_{i_{p+1}}^{p+1}\right) \ldots\left(\forall y_{1}^{n}\right) \ldots\left(\forall y_{i_{n}}^{n}\right)\left(T^{\prime}=U^{\prime}\right)
$$

формулы (8).

Считая значения $k_{1}, \ldots, k_{m}, i_{1}, \ldots, i_{p}$ фиксированными, а формулу (13) отличной от тождественно ложной и тождественно истинной формулы, обозначим через $K_{2}$ конъюнкцию равенств вида (10), которая по доказанному соответствует формуле (13). Элиминируем теперь в формуле

$$
\left(\exists y_{1}^{1}\right) \ldots\left(\exists y_{i_{1}}^{1}\right) \ldots\left(\exists y_{1}^{p}\right) \ldots\left(\exists y_{i_{p}}^{p}\right) K_{2}
$$

кванторы существования. Для этого воспользуемся хорошо известными эквивалентностями:

$$
\begin{array}{r}
\left(\exists v_{1}\right)\left(v_{1}=\cdots=v_{d}\right) \equiv\left\{\begin{array}{l}
\text { И, если } d=2, \\
v_{2}=\cdots=v_{d}, \quad \text { если } d>2,
\end{array}\right. \\
\left(\exists v_{1}\right)\left(v_{1}=\cdots=v_{d}=a\right) \equiv\left\{\begin{array}{l}
И, \\
\text { если } d=1, \\
v_{2}=\cdots=v_{d}=a, \quad \text { если } d>1 .
\end{array}\right.
\end{array}
$$


В результате получим, что каждая формула вида (14) либо тождественно истинна, либо эквивалентна бескванторной формуле, представляющей собой конъюнкцию формул вида $(10)$, где $v_{1}, \ldots, v_{d}$ - переменные типа $x_{q}^{s}$. Остается для заданных $x_{1}, \ldots, x_{m}$ проверить истинность каждой из таких формул и в соответствии со строением формулы (8) вычислить дизъюнкцию полученных $l_{1} \cdot \ldots \cdot l_{p}$ истинностных значений.

Сквозной просмотр описанного выше алгоритма показывает, что полиномиальное возрастание длин обрабатьваемых формул и соответственно полиномиальные затраты на время вычисления происходят дважды (см. структуру формулы (8)): при переходе от формул (12) к формулам (13) и при переходе от формул (14) к исходной формуле (8). Теорема доказана.

Перейдем к рассмотрению рудиментарных предикатов с произвольной кванторной приставкой. Пусть предикат $\rho\left(x_{1}, \ldots, x_{m}, z_{1}, \ldots, z_{n}\right)$ определяется формулой (4). Для всякой переменной $y_{j}$, для которой формула (4) содержит квантор существования по $y_{j}$, введем функцию

$$
f_{j}\left(x_{1}, \ldots, x_{m}, z_{1}, \ldots, z_{n}, y_{j 1}, \ldots, y_{j r_{j}}\right)
$$

где $y_{j 1}, \ldots, y_{j r_{j}}$ - все переменные из множества $\left\{y_{1}, \ldots, y_{n}\right\}$, которые в формуле (4) связаны кванторами обшности, предшествуюшими квантору $\exists y_{j}$. Функции (15) носят название скулемовских разрешаюших функиий (см., например, [8]); переменные $x_{1}, \ldots$, $x_{m}, z_{1}, \ldots, z_{n}$ в формулах (15) у нас играют роль параметров.

Функции (15) по определению обладают следуюшим свойством. Если при выбранных значениях $x_{1}, \ldots, x_{m}, z_{1}, \ldots, z_{n}$ значение $\rho\left(x_{1}, \ldots, x_{m}, z_{1}, \ldots, z_{n}\right)$ истинно, то для любых значений переменных $y_{k}$, стоящих в формуле (4) под кванторами общности, функции (15) дают такие значения переменных $y_{j}$, которые вьполняют формулу $T=U$. При этом, разумеется, как значения переменных $y_{k}$, так и значения функций (15) подчиняются ограничениям на длины диадических представлений, имеюшихся в формуле (4). Отметим еще, что функции (15), рассматриваемые от всех переменных $x_{1}, \ldots, x_{m}, z_{1}, \ldots$, $z_{n}, y_{j 1}, \ldots, y_{j} r_{j}$, являются, вообще говоря, частичными: мы не требуем определения их значений в том случае, когда значение $\rho\left(x_{1}, \ldots, x_{m}, z_{1}, \ldots, z_{n}\right)$ ложно.

Рудиментарный предикат $\rho\left(x_{1}, \ldots, x_{m}, z_{1}, \ldots, z_{n}\right)$, представимьй в форме $(4)$, назовем компактным, если для него возможен выбор таких скулемовских функций (15), которые обладают следуюшим свойством: если наборы

$$
\left(a_{1}, \ldots, a_{m}, b_{1}, \ldots, b_{n}, c_{1}, \ldots, c_{r_{j}}\right), \quad\left(a_{1}, \ldots, a_{m}, b_{1}, \ldots, b_{n}, c_{1}^{\prime}, \ldots, c_{r_{j}}^{\prime}\right)
$$

входят в область определения функции (15) и

$$
\left|c_{1}\right|=\left|c_{1}^{\prime}\right|, \ldots,\left|c_{r_{j}}\right|=\left|c_{r_{j}}^{\prime}\right|
$$

то

$$
\left|f_{j}\left(a_{1}, \ldots, a_{m}, b_{1}, \ldots, b_{n}, c_{1}, \ldots, c_{r_{j}}\right)\right|=\left|f_{j}\left(a_{1}, \ldots, a_{m}, b_{1}, \ldots, b_{n}, c_{1}^{\prime}, \ldots, c_{r_{j}}^{\prime}\right)\right| \cdot
$$

ТЕОРема 2. Любой компактный рудиментарный предикат можно вычислить подходящим детерминированным алгоритмом за полиномиальное время. 
ДокАЗАТЕЛьСТво. Пусть рудиментарньй предикат $\rho\left(x_{1}, \ldots, x_{m}, z_{1}, \ldots, z_{n}\right)$ представим формулой (4) и для него существуют скулемовские разрешаюшие функции (15), которые удовлетворяют сформулированному вьше условию компактности. Как и в теореме 1 , будем пользоваться обозначениями (6). Зафиксируем значения $k_{1}, \ldots, k_{m}, l_{1}$, $\ldots, l_{n}, i_{1}, \ldots, i_{n}$, где $1 \leqslant i_{1} \leqslant l_{1}, \ldots, 1 \leqslant i_{n} \leqslant l_{n}$. Попытаемся выяснить, может ли при заданных значениях $x_{1}, \ldots, x_{m}, z_{1}, \ldots, z_{n}$, удовлетворяюших соотношениям (6), вьполняться часть формулы (4), когда переменные $y_{d}$, стоящие в формуле (4) под кванторами обшности, принимают значения из множеств $\left\{y_{d}:\left|y_{d}\right|=i_{d}\right\}$, а значения переменных $y_{j}$, даваемые функциями (15), принадлежат множествам $\left\{y_{j}:\left|y_{j}\right|=i_{j}\right\}$. Для этого так же, как и в теореме 1, введем двоичные переменные

$$
x_{1}^{s}, \ldots, x_{k_{s}}^{s}, y_{1}^{t}, \ldots, y_{i_{t}}^{t}, \quad 1 \leqslant s \leqslant m, \quad 1 \leqslant t \leqslant n,
$$

заменим в термах $T, U$ переменные $x_{s}, y_{t}$ блоками $x_{1}^{s} \ldots x_{k_{s}}^{s}$ и $y_{1}^{t} \ldots y_{i_{t}}^{t}$ и обозначим полученные после этой замены термы через $T^{\prime}$ и $U^{\prime}$. Если $\left|T^{\prime}\right|=\left|U^{\prime}\right|$, то сравним далее в термах $T^{\prime}, U^{\prime}$ символы, стояшие на одинаковых позициях, образуем систему равенств $W$ вида (9) и затем транзитивное замыкание системы равенств $W$. Если транзитивное замькание не содержит противоречивых равенств, то приходим к конъюнкции $K_{1}$ равенств вида (10), которая эквивалентна формуле $T^{\prime}=U^{\prime}$.

Чтобы теперь дать ответ на поставленный выше вопрос, необходимо проверить, могут ли (при фиксированных значениях переменных $x_{1}, \ldots, x_{m}$ ) переменные $y_{1}^{j}, \ldots, y_{i_{j}}^{j}$, выражающие значения функции $(15)$, быть в конъюнкции $K_{1}$ функциями от переменных $y_{1}^{c}, \ldots, y_{i_{c}}^{c}$, где $y_{c} \in\left\{y_{j 1}, \ldots, y_{j r_{j}}\right\}$. Считая, что при заданных значениях переменных $x_{1}^{1}, \ldots, x_{k_{1}}^{1}, \ldots, x_{1}^{m}, \ldots, x_{k_{m}}^{m}$ в конъюнкции $K_{1}$ противоречивых равенств не образуется, замечаем, что сформулированное требование будет вьполняться, если любое равенство конъюнкции $K_{1}$, в которое входит переменная типа $y_{q}^{j}$, где переменная $y_{j}$ связана в формуле (4) квантором существования, не содержит:

1) переменной типа $x_{p}^{s}$ и переменной типа $y_{r}^{t}$, где переменная $y_{t}$ связана в формуле $(4)$ квантором обшности;

2) константы 1 или 2 и переменной типа $y_{r}^{t}$, где переменная $y_{t}$ связана в формуле (4) квантором обшности;

3) двух различных переменных типа $y_{r}^{t}$, где переменная $y_{t}$ связана в формуле (4) квантором обшности;

4) переменной типа $y_{r}^{t}$, где переменная $y_{t}$ связана в формуле (4) квантором общности, следуюшим в кванторной приставке за квантором $\exists y_{j}$.

Таким образом, чтобы выяснить, может ли при заданных значениях $x_{1}, \ldots, x_{m}$, $z_{1}, \ldots, z_{n}$, удовлетворяюших соотношениям (6), выполняться часть формулы (4), когда переменные $y_{d}$, связанные в формуле (4) кванторами обшности, принимают значения из множеств $\left\{y_{d}:\left|y_{d}\right|=i_{d}\right\}$, необходимо провести описанную вьше проверку "на функциональность", задавая переменным $y_{j}$, которые связаны в формуле (4) кванторами существования, всевозможные области изменения $\left\{y_{j}:\left|y_{j}\right|=i_{j}\right\}, 1 \leqslant i_{j} \leqslant l_{j}$. Очевидно, на этом этапе будет затрачено полиномиальное (относительно длин диадических представлений $\left.x_{1}, \ldots, x_{m}, z_{1}, \ldots, z_{n}\right)$ время. Полностью процесс вычисления значения $\rho\left(x_{1}, \ldots, x_{m}, z_{1}, \ldots, z_{n}\right)$ состоит в выполнении предыдущего этапа при всевозможном выборе областей изменения $\left\{y_{d}:\left|y_{d}\right|=i_{d}\right\}, 1 \leqslant i_{d} \leqslant l_{d}$, для переменных $y_{d}$, связанных в формуле (4) кванторами общности. Теорема доказана. 
ЗАмЕчАнИЕ. Хотя теорема 2 имеет вид положительного утверждения, ее значение имеет скорее отрицательную направленность. Как показывают результаты работ [5], [7], маловероятно, чтобы любой рудиментарный предикат можно было выгислить детерминированным алгоритмом за полиномиальное время. Поэтому теорему 2 можно рассматривать как довод в пользу того, что не все рудиментарные предикаты являются компактными.

\section{СПИСОК ЦИТИРОВАННОЙ ЛИТЕРАТУРЫ}

[1] Кузнецов А. В.К теореме о канонической форме для ординальнорекурсивных функций // Гудстейн Р. Л. Математическая логика. М.: ИЛ, 1961. С. 149-154.

[2] Смальян Р. Теория формальных систем. М.: Наука, 1981.

[3] Непомнящий В.А. Рудиментарные предикаты и тьюринговы вычисления // Докл. АН CCCP. 1970. T. 195. № 2. C. 282-284.

[4] Непомнящий В.А. Рудиментарная интерпретация двуленточных тьюринговых вычислений // Кибернетика. 1970. №2. С. 29-35.

[5] Непомнящий В. А. Рудиментарное моделирование недетерминированных тьюринговых вычислений // Кибернетика. 1973. № 2. С. 23-29.

[6] Косовский Н. К. Элементы математической логики и ее приложения к теории субрекурсивных алгоритмов. Ленинград: Из-во Ленинградского ун-та, 1981.

[7] Марченков С. С. О сложности вычисления рудиментарных предикатов // Дискретная матем. 2000. Т. 12. № 4. С. 83-98.

[8] Ершов Ю. Л., Палютин Е. А. Математическая логика. М.: Наука, 1979.

Московский государственный университет 